\title{
Impact of manual uterine displacement and left lateral tilt on echocardiographical velocity time intergral (stroke volume) in advanced pregnancy
}

\author{
K A Knipe, ${ }^{1} \mathrm{BSc}, \mathrm{MB} \mathrm{ChB} ; \mathrm{E}$ J Langenegger, ${ }^{1} \mathrm{MB}$ ChB, FCOG (SA), MMed (O\&G), PhD; \\ W Muller, ${ }^{2}$ MB ChB, FCP (SA), MMed (Int Med); P Herbst, ${ }^{2}$ MB ChB, MRCP (UK), FCP (SA), Cert Cardiol (SA) \\ ${ }^{1}$ Department of Obstetrics and Gynaecology, Tygerberg Hospital and University of Stellenbosch, Cape Town, South Africa \\ ${ }^{2}$ Department of Medicine, Tygerberg Hospital and University of Stellenbosch, Cape Town, South Africa
}

Corresponding author: K A Knipe (karusha30@gmail.com)

Background. Pregnant women undergo a variety of physiological changes, and cardiac output (CO) is one of these important changes. During advanced pregnancy, aortocaval compression decreases CO by up to $30-40 \%$. Such compression may cause adverse physiological disturbances in both the mother and the baby. Current practice is to nurse pregnant woman with at least $15^{\circ}$ lateral tilt. Most studies have used non-invasive blood pressure measurement, which is not the gold standard and does not give a true depiction of the cardiac response to change in position.

Objective. To determine the impact of manual uterine displacement (MUD) on cardiac stroke volume (SV) using the current most accurate non-invasive method, i.e. descending aorta velocity time integral (VTI) with echocardiography (ECHO). This method was used to ascertain the appropriate position to improve $\mathrm{CO}$ and provide superior uterine perfusion.

Methods. A prospective case crossover study consisting of 22 women at 36 - 40 weeks of gestation was conducted. The studied positions were supine, $30^{\circ}$ tilt, left lateral tilt and manual uterine displacement. Patients received a screening ECHO to ensure normal cardiac structure and function followed by measurement of the descending aorta VTI at each of the positions with 2-minute intervals between position changes.

Results. There was a significant increase in the aortic VTI with all changes in position. The mean (standard deviation (SD)) change for supine-left lateral was $3.68(0.74)(p=0.00)$, supine-MUD was $3.29(0.72)(p=0.00)$ and supine- $30^{\circ}$ was $2.93(0.72)(p=0.00)$. No significant difference was found when the positions were compared with each other.

Conclusion. Lateral tilt and MUD significantly increased descending aorta VTI (SV).

S Afr J Obstet Gynaecol 2020;26(2):60-64. https://doi.org/10.7196/SAJOG.2020.v26i2.1603

The pregnant woman undergoes a variety of physiological changes, and cardiac output $(\mathrm{CO})$ is an important one. $\mathrm{CO}$ is the product of stroke volume (SV) and heart rate (HR), where stroke volume is the volume of blood ejected by the heart per beat and is normally $70 \mathrm{~mL} \cdot{ }^{[1,2]}$ During pregnancy, plasma volume increases from the non-pregnant level of $2600 \mathrm{~mL}$ to about $3800 \mathrm{~mL}$. CO rises by about $40 \%$ from $4.5-6 \mathrm{~L} / \mathrm{min}$ and this rise reaches a plateau at about 24 - 30 weeks' gestation. This is maintained throughout labour and returns to pre-pregnancy levels at variable times post delivery. During advanced pregnancy, aortocaval compression occurs as the uterus compresses the aorta and inferior vena cava, or both. This phenomenon has been described as early as 1959. ${ }^{[3]}$ Although initially thought to be a problem confined to the supine position, aortocaval compressions have also been shown in semi recumbent positions such as $15-30^{\circ}$ lateral tilt. ${ }^{[1,2,4]}$ As a consequence, CO may decrease by as much as $30-40 \%$, causing what is known as supine hypotension. ${ }^{[5]}$ Current global practice is to nurse pregnant woman with at least $15^{\circ}$ lateral tilt. It has been described in the literature that relief of aortocaval compression during anaesthesia, labour and fetal resuscitation plays a vital role in preventing supine hypotension and allowing adequate venous return to maintain SV and CO while awaiting caesarean section (CS). ${ }^{[1,2,4-6]}$ However, many studies in the literature used non-invasive blood pressure (BP) mechanisms or electrical cardiometry, which are not considered the gold standard to measure cardiac response to change in position. A recent study looked at the effect of various degrees of lateral tilt on aortocaval volume using magnetic resonance imaging (MRI), and showed that no degree of tilt changed aortic volume and inferior vena cava volume was only increased with tilt of $30^{\circ}$ or more ${ }^{[7]}$ A systematic review looking at 11 studies found that there is limited evidence to support or disprove the value of the use of lateral tilt and that MUD may be better than left lateral tilt but more data are needed to confirm these findings. ${ }^{[2]}$ The MUD-technique allows for relief of the aortocaval compression while the mother is supine (Fig. 1). This manoeuvre is useful when the mother cannot be turned onto her left side, e.g. during cardiopulmonary resuscitation $(\mathrm{CPR})$ or intubation. ${ }^{[2,8]}$

Currently, the most accurate non-invasive measurement of SV is provided by the VTI using ECHO. ${ }^{[9]}$ VTI is measured by tracing a pulse wave velocity Doppler profile. $\mathrm{CO}$ is then calculated as VTI $\times$ the cross-sectional area (CSA) of the structure where the VTI was measured (typically aorta or left ventricular outflow tract (LVOT)). Because the CSA has been demonstrated to remain relatively constant in the same patient over multiple measurements, 
the VTI is an excellent modality for assessing change in CO over multiple measurements. Furthermore, the Doppler ultrasound tool used to provide this measurement has been shown to be safe in pregnancy. ${ }^{[10]}$

Our study investigated the impact of MUD, $30^{\circ}$ left lateral tilt and $90^{\circ}$ left lateral tilt on ECHO VTI (SV) in advanced pregnancy.

\section{Methods}

A prospective, case crossover study was conducted in the Department of Obstetrics and Gynaecology at Tygerberg Hospital and the University of Stellenbosch. Pregnant women from the antenatal clinic were prospectively enrolled between February - April 2019. A consecutive sampling technique was applied from a cohort of the antenatal obstetric clinic. For inclusion, women had to have reached the gestational age range of 36 - 40 weeks and have normal resting ECHO. Exclusion criteria included chronic medical conditions, previous adverse pregnancy outcomes, current or previous complicated pregnancy and a body mass index (BMI) $<45$. $\mathrm{ECHO}$ was performed based on the minimum dataset as required by the British Society of Echocardiography. Any women with cardiac abnormalities were worked up further in the combined obstetric and cardiac high-risk clinic and excluded from the study. Blood pressure, heart rate, haemoglobin and estimated fetal weight were recorded at inclusion. The women then received regular antenatal check-ups and management of pregnancy.

Each echocardiogram was done at the combined obstetric and cardiac high-risk clinic with maternal fetal medicine specialist (EL) and cardiologist (WM) present. All the echocardiograms were done by the same operator (WM) using the General Electric Vivid S6 with 3S TTE (transthoracic ECHO) probe (General Electric, USA).

A baseline (supine) VTI measurement using continuous and pulse wave Doppler was obtained. VTI measurements in the proximal descending aorta were obtained from a suprasternal view. This view achieves the best Doppler alignment to flow in all study positions. Measurements were done with the women placed in the supine, $30^{\circ}$ (using a $30^{\circ}$ cushioned wedge), $90^{\circ}$ left lateral positions and then supine with gentle MUD performed by the same investigator (KK) for each woman. An aortic VTI was measured at each position after a 2-minute interval following a position change. VTI was obtained from a suprasternal view for the aorta with the probe in the suprasternal notch and the marker facing 1 o'clock position, perfectly aligned with aortic flow to minimise error. Measurements were taken $\sim 1 \mathrm{~cm}$ distal to the aortic arch (Fig. 2).

Enrolled women acted as their own controls. Measurements were compared in various positions on the same woman and each change was also compared with baseline (supine position). To improve statistical power, a paired measurement analysis was employed. ${ }^{[11]}$ A sample size of at least 20 women, each serving as her own control was thus calculated based on a change of $13 \%$ in the aortic VTI, which is accepted as a clinically significant change in the stroke volume based on previous literature. ${ }^{[12]}$

Data were populated onto Microsoft Excel spreadsheets and analysed using STATA software version 14 (StataCorp., USA). Comparison between groups was made by paired ANOVA with post hoc adjustments (Bonferroni). Normal distribution of data was determined using histograms with skewness $-1.1-1.1$ and Kurtosis $-2.2-2.2$. Median and mean values did not differ by $>1$ for any of the data sets. Any data that did not meet criteria for normal distribution were described using median and interquartile range (IQR).

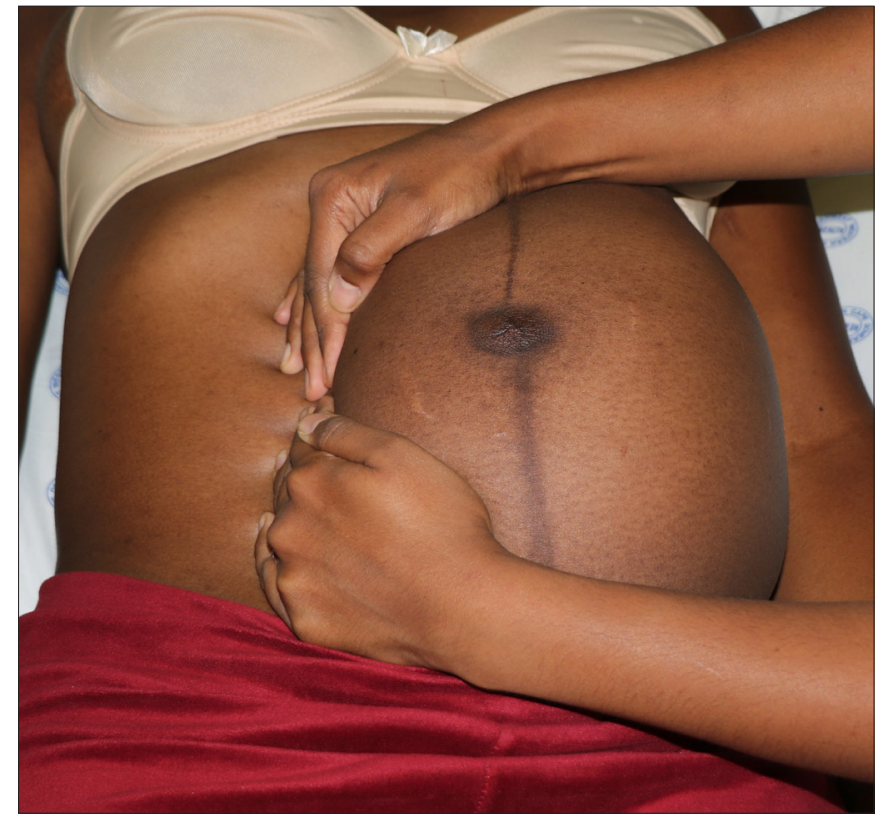

Fig. 1. Manual uterine displacement (MUD) technique.

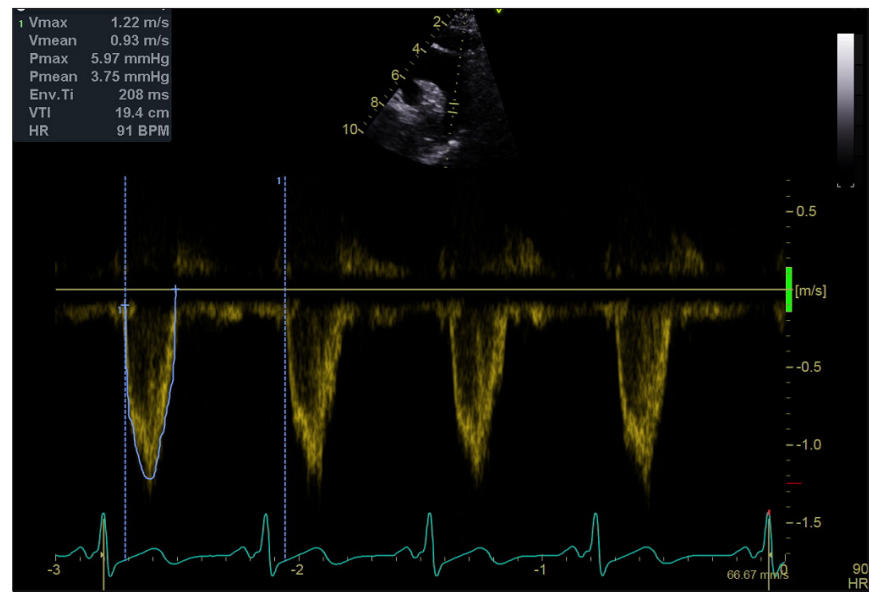

Fig. 2. Echocardiogram of suprasternal view demonstrating the measurement of descending aortic VTI.(VTI = velocity time integral. $)$

Ethics approval was obtained from Stellenbosch University Health Research Ethics Committee (ref. no. S18/06/119) and the National Health Research Ethics Council (NHREC) (ref. no. REC-130408-012 and-REC-230208-010). Informed consent was obtained from each participant.

\section{Results}

A total number of 24 women met inclusion criteria but two were excluded, based on baseline echocardiographical findings. The study population demographics are summarised in Table 1. All baseline monitoring was within normal range.

All women received a baseline echocardiogram which showed normal structure and function. The values of variables measured are summarised in Table 2.

The descending aorta VTI was measured in the 3 positions and results are summarised in Table 3.

The descending aorta VTI showed a significant increase with $30^{\circ}$, $90^{\circ}$ left lateral and MUD compared with supine position. The mean (SD) change for the supine position to left lateral was $3.68(0.7)$ $(p<0.001)$, supine position with MUD was $3.29(0.72)(p<0.001)$ and 
Table 1. Study population demographics

\begin{tabular}{ll}
\hline Characteristics & Mean $(\mathrm{SD})^{\star}$ \\
\hline Age (years) & $30.2(5.7)$ \\
Gravidity, median (IQR) & $3(1)$ \\
Parity, median (IQR) & $2(1)$ \\
BMI & $35(6.65)$ \\
Gestation (weeks) & $37(1.46)$ \\
SBP (mmHg) & $114.68(9.58)$ \\
DBP (mmHg) & $60.45(6.1)$ \\
$\mathrm{HR}(\mathrm{bpm})$ & $80.31(13.82)$ \\
EFW (grams) & $3052(327.92)$ \\
$\mathrm{Hb}$ & $11.39(1.55)$ \\
SD = standard deviation; IQR = interquartile range; BMI = body mass index; SBP = systolic \\
blood pressure; DBP = diastolic blood pressure; HR = heart rate; $\mathrm{EFW}=$ estimation of fetal \\
weight; $\mathrm{H}$ = haemoglobin. \\
${ }^{*}$ Unless otherwise specified.
\end{tabular}

Table 2. Baseline echocardiographical findings

\begin{tabular}{|c|c|c|}
\hline ECHO measurement & Mean $(\mathrm{SD})^{\star}$ & Normal range \\
\hline $\mathrm{RV}$ area $\left(\mathrm{cm}^{2}\right)$ & $14.4(3,45)$ & $<25$ \\
\hline IVSD $(\mathrm{cm})$, median (IQR) & $0.8(0.2)$ & $0.6-1.1$ \\
\hline $\operatorname{LViD}(\mathrm{cm})$ & $4.36(1.03)$ & $3.9-5.3$ \\
\hline LVPWd (cm) & $0.76(0.13)$ & $0.6-1.2$ \\
\hline LViDs (cm) & $3.2(0.37)$ & $3.2-5.3$ \\
\hline LVPWs (cm) & $0.83(0.15)$ & $0.6-1.2$ \\
\hline LVOT diameter $(\mathrm{cm})$ & $2.06(0.14)$ & $1.85-2.35$ \\
\hline Ejection fraction (\%) & $61.89(5.9)$ & $>55$ \\
\hline LA diameter $(\mathrm{cm})$ & $3.11(0.51)$ & $2.7-3.8$ \\
\hline RV diameter $(\mathrm{cm})$ & $2.85(0.51)$ & $<4.2$ \\
\hline \multicolumn{3}{|c|}{ 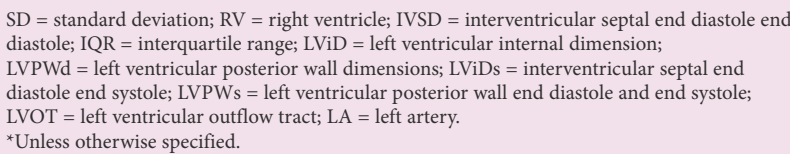 } \\
\hline
\end{tabular}

Table 3. VTI comparison in different positions

\begin{tabular}{|c|c|c|c|c|}
\hline & Supine & MUD & Left lateral & $30^{\circ}$ wedge \\
\hline Mean (SD) & $20.9(4.96)$ & $24.19(4.63)$ & $24.58(4.99)$ & $23.83(4.83)$ \\
\hline $\begin{array}{l}\text { Change } \\
\text { compared with } \\
\text { supine (SE) }\end{array}$ & - & $3.29(0.72)$ * & $3.68(0.75)$ * & $2.93(0.72) *$ \\
\hline$\%$ change & - & 22 & 22 & 19 \\
\hline
\end{tabular}

supine with wedge was $2.93(0.72)(p=0.001)$. This is graphically represented in Figs 3 and 4.

All positions showed a statistically significant increase in VTI when compared with the supine position and no significant difference between the 3 positions was found when compared with each other.

\section{Discussion}

This study demonstrated that left lateral tilt as well as MUD increased SV and CO (Fig. 4). An average increase in VTI with MUD was $3.29 \mathrm{~cm}$ using the formulae $\mathrm{SV}=\pi \mathrm{r}^{2} \times \mathrm{VTI}$, with a normal aortic radius in the adult female being $1.16 \mathrm{~cm},{ }^{[13]}$ and this translated to an average increase of $13.9 \mathrm{~mL}$ in SV. Therefore, CO increased by $1112 \mathrm{~mL} / \mathrm{min}(1.1 \mathrm{~L} / \mathrm{min})$ at an average $\mathrm{HR}$ of 80 for the study population $(\mathrm{CO}=\mathrm{SV} \times \mathrm{HR})$.

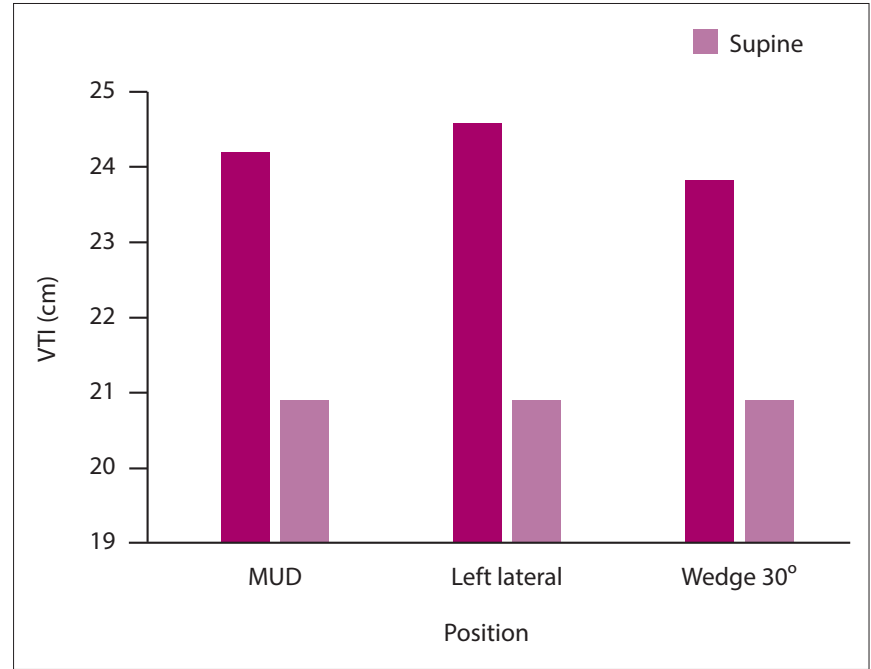

Fig. 3. Absolute mean VTI (cm) compared with baseline (supine). measurement of descending aortic VTI. $(V T I=$ velocity time integral. $)$

There was an increase of $15.5 \mathrm{~mL}$ in SV with $90^{\circ}$ left lateral and therefore an increase of $1.2 \mathrm{~L} / \mathrm{min}$ in $\mathrm{CO}$. Hence, with an average $\mathrm{CO}$ of $6 \mathrm{~L} / \mathrm{min}$ at term, removal of aortocaval compression by means of tilt/MUD will provide a $18-20 \%$ increase in CO. There are no studies reported in the literature that assessed SV in supine compared with MUD using the aortic VTI.

Humphries et al. ${ }^{[14]}$ found similar results when looking at CO using MRI and showed a $16.4 \%$ increase in CO from supine to left lateral position. They also showed that in late pregnancy, women had significant collateral venous blood flow owing to the extent of the IVC compression. This could account for other studies not showing the same positional effect. However, in a compromised haemodynamic state (e.g. shock, maternal cardio-respiratory arrest), the collateral venous supply may not be enough to maintain adequate CO.

Another possible explanation for the conflicting opinions regarding the benefit of tilt could be due to the paucity of evidence regarding fluid status. Fluid status is one of the many variables that have a significant impact on CO changes. This challenge was overcome in all of our women by having normal baseline ECHO as well as them acting as their own controls. This ensured that regardless of the fluid status, the fluid status would remain constant with comparative position changes, thereby strengthening the changes observed.

There is a wide variety of information published regarding the value of different degrees of tilt and the value of uterine displacement in pregnant women. However, there is a lack of consensus as to whether it is beneficial for improving $\mathrm{CO}$, and this was the rationale for this study.

Some anaesthetic studies have investigated and shown benefit of at least $15^{\circ}$ tilt in improving CO ${ }^{[1,12,15]}$ This benefit, however, was only shown in boar and not in women who are not under anaesthesia. ${ }^{[1]}$ This study also did not use ECHO for evaluation of the change but rather used the ultrasonic cardiac output monitor, which is a less accurate modality.

Pregnant women are still nursed with at least $15^{\circ}$ left lateral tilt due to the presumed fetal benefits, globally. ${ }^{[14]}$ A randomised control trial showed no difference in neonatal outcome or acidbase status in CS done in supine v. tilt positions. ${ }^{[15]}$ Other studies have shown benefits of a lateral tilt position compared with the supine position in improving umbilical venous oxygenation. ${ }^{[16]}$ The 


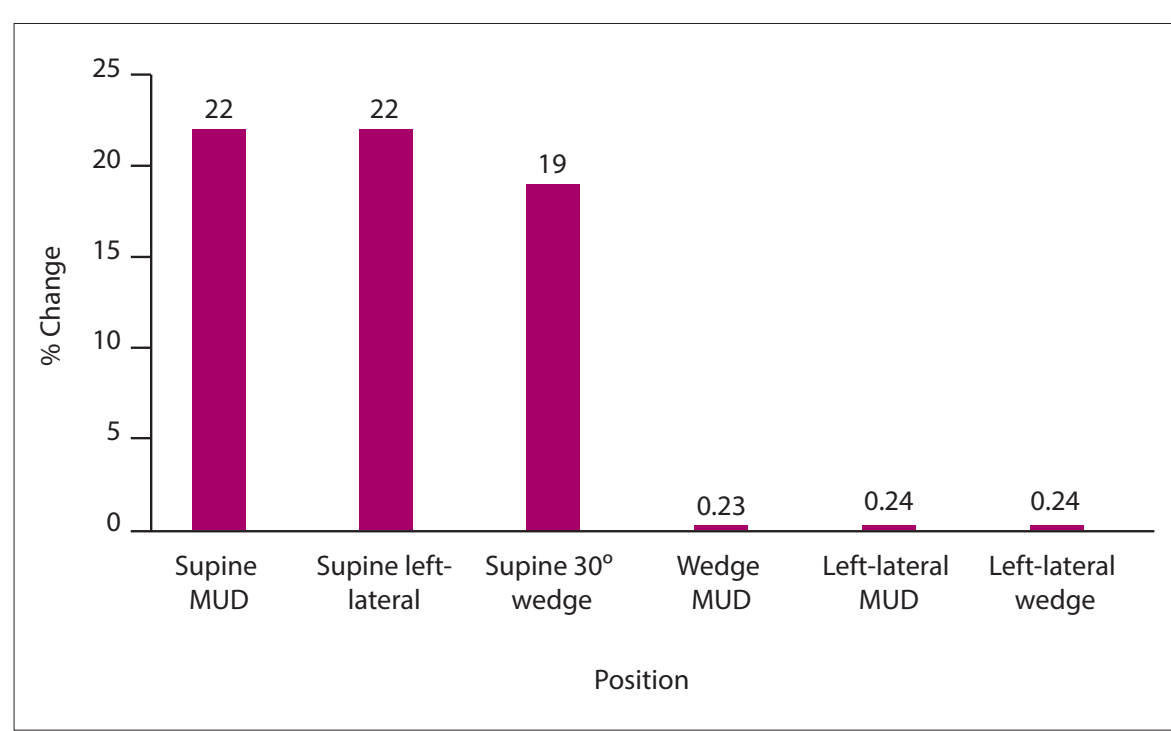

Fig. 4. Comparative change in $\%$ VTI. (VTI = velocity time integral. $)$

placenta depends on an adequate $\mathrm{CO}$ for its perfusion. There is currently no consensus in the literature as to the benefit of tilt, what degree of tilt provides benefit and whether this presumed benefit can be reproduced in the supine position using MUD.

It is challenging to achieve successful chest compressions in a pregnant woman on her left side during maternal resuscitation. ${ }^{[6]}$ It is also likely that chest compressions and attaining an effective $\mathrm{CO}$ will not be possible with unrelieved aortocaval compression. In the event that maternal cardio-respiratory arrest occurs, the idea of hysterotomy to deliver the fetus, relieving the aortocaval compression and allowing successful chest compressions in the supine position is the rationale for the perimortem CS. ${ }^{[6]}$ Previous studies have investigated various methods of tilt during CPR and found compressions most effective in the supine position while acknowledging the need to relieve aortocaval compression. ${ }^{[17]}$

We have shown that MUD is as effective a non-invasive manoeuvre at relieving aortocaval compression in the supine

position. The non-invasive manoeuvre can be added to any maternal resuscitation protocol to improve venous return, allowing more efficient chest compressions in the supine position during CPR prior to the need for perimortem CS. MUD has already been incorporated into a few maternal resuscitation guidelines. ${ }^{[18,19]}$ This study provides further scientific support for this practice and found an increase in SV/CO using a gold standard echocardiographic technique to compare relief of aortocaval compression in various positions.
MUD VTI measurement was done without shifting the woman from the baseline (supine) position, but only by gently pulling and lifting the uterus away from the midline (toward the left in this study) as far as possible without compromising her comfort. A similarly significant increase in VTI was demonstrated despite no change in the physical position. This implies that an increase in SV/CO is secondary to the relief of aortocaval compression rather than due to the position itself. Although this study cannot assume proven clinical benefit, an increase in SV/CO of $20 \%$ is considered haemodynamically significant. ${ }^{[12]}$

\section{Study limitations and strengths}

The limitations of this study include the fact that operators were not blinded in terms of the positioning of the women at the time of image acquisition. However, bias was minimised by having the study analysis and measurement done at a later stage by a senior cardiologist who was not present at time of positioning. An additional limitation could be related to the angulation of the probe during position changes but care was taken to carefully check the direction of aortic flow prior to measurement being taken.

A strong point of this study is the fact that women acted as their own control therefore, eliminating confounding bias. Additionally, due to the fact that we measured the direct column of blood passing a particular point in the same vessel, in the same woman, at the same gestation, this reduced the likelihood that other systemic responses contributed to the outcome.

\section{Conclusion}

Left lateral tilt and MUD significantly increased SV/CO when compared with the supine position. MUD and tilt, be it $30^{\circ}$ or full left lateral $\left(90^{\circ}\right)$, did not show a significant difference when compared with each other and therefore can be used interchangeably depending on the clinical scenario and patient comfort without compromising patient benefit. MUD can be used to increase SV during maternal resuscitation in the supine position.

Declaration. This study was done in partial fulfilment of a MMed degree.

Acknowledgements. We would like to thank $\mathrm{Mr}$ Michael McCaul from the Division of Epidemiology and Biostatistics at the Department of Global Health at Stellenbosch University for assisting with the statistical analysis of data. The Department of Obstetrics and Gynaecology at Tygerberg Hospital for enabling the progress of this study as well as the Department of Cardiology for the skills and time offered by members of the team to review images and assist with echocardiography. Author contributions. KK, EL and $\mathrm{PH}$ conceptualised the study, developed the protocol, recruited patients, supervised and assisted with data collection and wrote the manuscript. WM performed the echocardiographs necessary for the study. All authors approved the manuscript for publication.

Funding. None.

Conflicts of interest. None.

1. Hasanin A, Soryal R, Kaddah T, et al. Hemodynamic effects of lateral tilt before and after spinal anesthesia during cesarean delivery: An observational study. BMC Anesthesio 2018;18(1):4-9. https://doi.org/10.1186/s12871-018-0473-0

2. Cluver C, Novikova N, Hofmeyr GJ, Hall DR. Maternal position during caesarean section for preventing maternal and neonatal complications. Cochrane Database Syst Re 2013;March 28(3). https://doi.org/10.1002/14651858 cd007623.pub3

3. Pyorala T, Pyorala K. Supine hypotensive syndrome in late pregnancy. Duodecim 1959;75:689-698.

4. Kinsella SM, Whitwam JG, Spencer JAD. Reducing aortocaval compression: How much tilt is enough? aortocaval compression: How much tilt is enough?
$\mathrm{Br}$ Med J 1992;305(6853):539-540. https://doi. org/10.1136\%2Fbmj.305.6853.539

5. Chesnutt AN. Physiology of normal pregnancy. Crit Care Clin 2004;20(4):609-615. https://doi:10.1016/j/ccc.2004.06.001

6. Katz VL. Perimortem caesarean delivery: Its role in maternal mortality. Semin Perinatol 2012;36(1):68-72. https://doi. mortality. Semin Perinatol 2012,3
org/10.1053/j.semperi.2011.09.013

7. Higuchi H, Takagi S, Zhang K, Furui I, Ozaki M. Effect of lateral tilt angle on the volume of the abdominal aorta and inferior vena cava in pregnant and nonpregnant women determined by magnetic resonance imaging. Anesthesiology 2015;122(2):286-293. https://doi.org/10.1097/ aln. 0000000000000553

8. Augustin G. Abdominal trauma. In: Acute Abdomen During Pregnancy. Cham: Springer International Publishing, 2014:325-400. https://doi.org/10.1007/978-3-319-05422-3_10

9. Petersen JW, Liu J, Chi YY, et al. Comparison of multiple non-invasive methods of measuring cardiac output during pregnancy reveals marked heterogeneity in the magnitude of cardiac output change between women. Physiol Rep 2017;5(8):1-11. https://doi.org/10.14814/phy2.13223 


\section{RESEARCH}

10. Liu S, Elkayam U, Naqvi TZ. Echocardiography in pregnancy: Part 1. Curr Cardiol Rep 2016;18(9):92. https://doi.org/10.1007/s11886-016-0760-7

11. Shintani WA. Sample size estimation and power computation on paired or skewed continuous data. GCRC Weekly Workshop. Department of Biostatistics; Vanderbilt University Medical Center 2006. http://biostat.mc.vanderbilt.edu/wiki/pub/Main/GCRCNoonWorkshops/ SampleSizeEstimationandPowerComputationonPaired.pdf

12. Dyer RA, Daniels A, Vorster A, et al. Maternal cardiac output response to colloid preload and vasopressor therapy during spinal anaesthesia for caesarean section in patients with severe preeclampsia: A randomised, controlled trial. Anaesthesia 2018;73(1):23-31. https://doi.org/10.1111/ anae. 14040

13. Atlas GM, Desiderio MC. Solutions to the Van der Pol equation: A model of aortic blood flow. Bioengineering Proceedings of the Northeast conference. 2006.143-144. https://doi:10.1109 NEBC.2006.1629793

14. Humphries A, Mirjalili SA, Tarr GP, Thompson JMD, Stone P. The effect of supine positioning on maternal hemodynamics during late pregnancy. J Matern Neonatal Med 2019;32(23):3923-3930. https://doi.org/10.1080/14767058.2018.1478958

15. Lee A, Corradini B, Wang S, Goodman SR, Smiley RM. Left lateral table tilt for elective cesarean delivery. Anesthesiology 2017;127(2):241-249. https://doi.org/10.1097/aln.0000000000001737
16. Bamber JH, Dresner M. Aortocaval compression in pregnancy: The effect of changing the degree and direction of lateral tilt on maternal cardiac output. Anaesth Analg 2003;97(1):256-258. https:// doi.org/10.1213/01.ane.0000067400.79654.30

17. Ip JK, Campbell JP, Bushby D, Yentis SM. Cardiopulmonary resuscitation in the pregnant patient A manikin-based evaluation of methods for producing lateral tilt. Anaesthesia 2013;68(7):694-699. https://doi.org/10.1111/anae.1218

18. Vanden Hoek TL, Morrison LJ, Shuster M, et al. Part 12: Cardiac arrest in special situations 2010 American Heart Association Guidelines for cardiopulmonary resuscitation and emergency cardiovascular care. Circulation. 2010;122:S829-S861. https://doi.org/10.1161/ circulationaha.110.971069

19. Jain V, Chari R, Maslovitz S, et al. Guidelines for the management of a pregnant trauma patient. J Obstet Gynaecol Canada 2015;37(6):553-571. https://doi.org/10.1016/S1701-2163(15)30232-2

Accepted 22 October 2020. 